GLASNIK MATEMATIČKI

Vol. 41(61)(2006), $141-157$

\title{
HYPERSPACES WITH EXACTLY TWO ORBITS
}

\author{
Sam B. Nadler, Jr. and Patricia Pellicer-Covarrubias
}

West Virginia University, USA and Universidad Nacional Autónoma de México, México

\begin{abstract}
Let $C(X)$ be the hyperspace of all subcontinua of a (metric) continuum $X$. It is known that $C(X)$ is homogeneous if and only if $C(X)$ is the Hilbert cube. We are interested in knowing when $C(X)$ is $\frac{1}{2}$-homogeneous, meaning that there are exactly two orbits for the action of the group of homeomorphisms of $C(X)$ onto $C(X)$. It is shown that if $X$ is a locally connected continuum or a nondegenerate atriodic continuum, and if $C(X)$ is $\frac{1}{2}$-homogeneous, then $X$ is an arc or a simple closed curve. We do not know whether an arc and a simple closed curve are the only continua $X$ for which $C(X)$ is $\frac{1}{2}$-homogeneous.
\end{abstract}

\section{INTRODUCTION}

A continuum is a nonempty compact connected metric space; a nondegenerate continuum is a continuum with more than one point.

For a continuum $X$ with metric $d$, the hyperspace $C(X)$ is the space of all subcontinua of $X$ with the Hausdorff metric $H_{d}$ ([IN] or [N1]).

For a positive integer $n$, a space is said to be $\frac{1}{n}$-homogeneous provided that the action on the space of the group of homeomorphisms of the space onto itself has exactly $n$ orbits (an orbit being the action of the homeomorphism group at a given point $x$ ). Thus, 1-homogeneous spaces are the more familiar homogeneous spaces.

There seem to be only two papers about $\frac{1}{2}$-homogeneity. Krasinkiewicz $[\mathrm{Kr}]$ proved the Sierpiński universal curve is $\frac{1}{2}$-homogeneous, and Patkowska

2000 Mathematics Subject Classification. 54B20, 54F15.

Key words and phrases. Arc, arc-like, atriodic, chainable, circle-like, connected im kleinen, continuum, finest monotone map, Hilbert cube, homogeneous, $\frac{1}{2}$-homogeneous, $\frac{1}{n}$ homogeneous, hyperspace, layers (tranches), locally connected, $n$-cell, $n$-fold hyperspace, $n$-fold symmetric product, orbit, property of Kelley, simple closed curve, simple triod, treelike, triod. 
$[\mathrm{P}]$ studied $\frac{1}{2}$-homogeneous compact ANR's of dimension $\leq 2$. In particular, our paper is the first paper to study $\frac{1}{2}$-homogeneous hyperspaces.

Our paper is partly motivated by the fact that it is known when $C(X)$ is homogeneous; namely, for nondegenerate continua $X, C(X)$ is homogeneous only when $C(X)$ is the Hilbert cube [N1, p. 564, 17.2]; in other words, only when $X$ is a locally connected continuum in which every arc is nowhere dense.

We are interested in determining all those continua $X$ for which $C(X)$ is $\frac{1}{2}$-homogeneous. The problem seems to be quite difficult to solve since there seem to be very few such continua. In fact, the only two we know of are an arc and a simple closed curve:

Proposition 1.1. If $X$ is an arc or a simple closed curve, then $C(X)$ is $\frac{1}{2}$-homogeneous.

Proof. In both cases, $C(X)$ is a 2-cell [IN, pp. 33-35, 5.1 and 5.2]; therefore, $C(X)$ is $\frac{1}{2}$-homogeneous (use Brouwer's Invariance of Domain Theorem [HW, p. 95, Theorem VI 9]).

Thus, we are concerned with the converse of Proposition 1.1:

Question. If $X$ is a continuum such that $C(X)$ is $\frac{1}{2}$-homogeneous, then is $X$ an arc or a simple closed curve? What about when $C(X)$ is finite dimensional?

We answer the question affirmatively for locally connected continua (Theorem 3.1) and for nondegenerate atriodic continua (Theorem 5.1). We also obtain results related to the question under the additional assumption that $C(X)$ is finite dimensional (Theorem 3.9 through Theorem 3.11); we note that we do not know if $C(X)$ must be finite dimensional when $C(X)$ is $\frac{1}{2}$ homogeneous (see Corollary 3.10 and Question 6.5).

We give several corollaries to Theorem 5.1. The corollaries single out various classes of continua that are of significant interest in continuum theory and hyperspace theory. The principal corollary answers the question we have asked for the class of all continua each of whose nondegenerate proper subcontinua is arc-like (Corollary 5.2). This class of continua includes arc-like continua, circle-like continua and certain important tree-like continua. We also give a generalization of the first corollary (Corollary 5.7).

In Section 6, we pose more questions (with comments) related to our results.

\section{Notation, terminology, AND PRELIminary RESUlts}

Most of our notation is standard; we only give notation that we feel is necessary to include: $I$ denotes the unit interval $[0,1]$. If $\Delta^{2}$ is a 2-cell, $i \Delta^{2}$ and $\partial \Delta^{2}$ denote the manifold interior and manifold boundary of $\Delta^{2}$, respectively. We use $\times$ in denoting Cartesian product. $\bar{A}$ denotes the closure of $A$. $\operatorname{dim}$ 
denotes topological dimension [HW]. For $A \in C(X), \mathcal{O}(A)$ denotes the orbit of $A$ in $C(X)$.

We assume the reader is familiar with basic ideas concerning continua and hyperspaces. For convenient refence, we summarize terminology and known results that are central to our paper and that, for the most part, we use several times; we also reference where proofs of the results can be found. First, we list some terminology; terminology not included in this section is in our introduction or in [IN], [N1] or [N2].

A continuum $X$ is connected im kleinen at a point $x$ provided that $x$ has arbitrarily small connected neighborhoods (not necessarily open in $X$ ).

The terms arc-like, circle-like and tree-like refer to continua that admit $\epsilon$-maps for each $\epsilon>0$ onto the interval $I$, the unit circle $S^{1}$, or a tree, respectively. These notions are sometimes defined in terms of covers and called chainable (or snake-like), circularly-chainable or tree-chainable, respectively (e.g., see [N2, p. 235, 12.11]).

A continuum $X$ is said to be irreducible provided that there are points $p, q \in X, p \neq q$, such that no proper subcontinuum of $X$ contains both $p$ and $q[\mathrm{~N} 2$, p. $68,4.35]$.

A subcontinuum $T$ of a continuum $X$ is said to be terminal in $X$ provided that if $A$ is any subcontinuum of $X$ such that $A$ intersects both $T$ and $X-T$, then $A \supset T$. Note that for any point $x \in X,\{x\}$ is terminal in $X$.

A continuum $X$ is a triod provided that for some subcontinuum $Z$ of $X$, $X-Z$ is the union of three nonempty mutually separated sets $[\mathrm{N} 2$, p. 208, 11.22]. A continuum $X$ is said to be atriodic provided that $X$ contains no triod [N2, p. 231, 12.3]. A weak triod is a continuum that is the union of three subcontinua, no one of which is contained in the union of the other two, and such that the intersection of all three is nonempty [N2, p. 209, 11.24].

Let $X$ be a continuum. An order arc in $C(X)$ is an $\operatorname{arc} \alpha$ in $C(X)$ that is a nest (i.e., if $A, B \in \alpha$, then $A \subset B$ or $B \subset A$ ); terminology is from [IN] and $[\mathrm{N} 1]$.

A continuum $X$ has the property of Kelley at a point $a \in X$ provided that for each $\epsilon>0$, there is a $\delta=\delta(a, \epsilon)>0$ such that if $b \in X, d(a, b)<\delta$ ( $d$ being the metric for $X$ ) and $a \in A \in C(X)$, then there exists $B \in C(X)$ such that $b \in B$ and $H_{d}(A, B)<\epsilon$ ([N1, p. 551, 16.23], where the property of Kelley is called property $[\kappa])$. A continuum has the property of Kelley if it has the property of Kelley at each point.

One of our principal tools is the use of layers (or tranches) of irreducible hereditarily decomposable continua. We refer the reader to [Ku, pp. 190-219] or $[\mathrm{T}]$ for the general theory of layers. The term layer is from $[\mathrm{Ku}]$ and means any element of the finest monotone upper semicontinuous decomposition of a continuum $Y$, where the decomposition space is an arc (see 2.1 below). A map $\varphi$ from $Y$ onto $I$ whose point inverses are the layers of $Y$ is called a finest monotone map. We call $\varphi^{-1}(0)$ and $\varphi^{-1}(1)$ end layers of $Y$. 
We note the following result about layers:

2.1. If $Y$ is an irreducible hereditarily decomposable continuum, then there is a unique finest monotone upper semicontinuous decomposition of $Y$ (hence, a (nonunique) finest monotone map of $Y$ onto I) whose quotient space is an arc, and each layer is nowhere dense in $Y([\mathrm{Ku}, \mathrm{p} .216$, Theorem 3] or [T, p. 15, Theorem 10], uniqueness is by [T, p. 10, Theorem 6]).

Our next result is commonly referred to as the Boundary Bumping Theorem.

2.2. Let $X$ be a continuum, and let $U$ be a nonempty, proper and open subset of $X$. Then every component of the closure $\bar{U}$ intersects $\bar{U}-U$ ([IN, p. 101, 12.10] or [N2, p. 73, 5.4]).

The remaining results in this section are known results about the hyperspace $C(X)$. The first two of the results concern arcwise connectivity.

2.3. If $X$ is a continuum and $A$ is a subcontinuum of $X$, then $C(X)-$ $C(A)$ is arcwise connected ([N1, p. 358, 11.2]).

2.4. For any continuum $X, C(X)$ is locally arcwise connected at $X$ ([N1, p. 154, 1.136] or [IN, p. 122, 15.5]).

Our next five results concern points of $C(X)$ that arcwise disconnect $C(X)$ (note that $C(X)$ is arcwise connected by [IN, p. 113, 14.9] or [N1, p. 65, $1.12])$.

2.5. A nondegenerate continuum $X$ is indecomposable if and only if the point $X$ of $C(X)$ arcwise disconnects $C(X)([\mathrm{N} 1, \mathrm{p} .104,1.51])$.

2.6. Let $X$ be a continuum such that $\operatorname{dim}(C(X))<\infty$, and let $Y$ be a nondegenerate subcontinuum of $X$. Then $Y$ is indecomposable if and only if $C(X)-\{Y\}$ has uncountably many arc components ([N1, pp. 314 and 357-358, $8.19,11.1$ and 11.2]).

In the next result, $K$ is not compact, but we use the natural notation $C(K)$ to denote the space of all subcontinua of $K$.

2.7. Let $X$ be a continuum and let $Y$ be a nondegenerate indecomposable subcontinuum of $X$. If $\mathcal{A}$ is an arc component of $C(X)-\{Y\}$ and $X \notin \mathcal{A}$, then there is a composant $K$ of $Y$ such that $\mathcal{A}=C(K)([\mathrm{N} 1, \mathrm{pp} .105$ and $358,1.52 .1$ and 11.2]).

2.8. Let $X$ be an atriodic continuum, and let $Y$ be a nondegenerate indecomposable subcontinuum of $X$. Then $C(X)-\{Y\}$ has uncountably many arc components (follows from the proof in [R, p. 286, Proposition 5]).

2.9. A nondegenerate proper subcontinuum of $X$ that is terminal in $X$ arcwise disconnects $C(X)([\mathrm{N} 1, \mathrm{p} .361,11.5])$.

Our final two results of this section concern singletons in $C(X)$. The second result is particularly important for the proofs of several of our results. 
2.10. A continuum $X$ is connected im kleinen at a point $x$ if and only if $C(X)$ is connected im kleinen at $\{x\}$ ([G, p. 391, Corollary 4]).

2.11. If $X$ is an atriodic continuum and $x \in X$, then $\{x\}$ does not belong to the manifold interior of any 2-cell in $C(X)$ (weakened form of $[\mathrm{A}, \mathrm{p} .40$, Theorem 3]).

\section{Preliminary Results about $\frac{1}{2}$-Homogeneous hyperspaces}

We use Lemma 3.5 and Lemma 3.7 in the proof of our main result, Theorem 5.1. In addition, we obtain results that are of interest independent of Theorem 5.1. Theorem 3.1 answers the question in Section 1 affirmatively for locally connected continua. Theorem 3.8 concerns any continuum $X$ whose hyperspace $C(X)$ is $\frac{1}{2}$-homogeneous. Theorem 3.9 through Theorem 3.12 are also of separate interest from Theorem 5.1 (see comments after the proof of Theorem 3.8).

Theorem 3.1. If $X$ is a locally connected continuum, then $C(X)$ is $\frac{1}{2}$ homogeneous if and only if $X$ is an arc or a simple closed curve.

Proof. If $X$ is an arc or a simple closed curve, then $C(X)$ is $\frac{1}{2}$ homogeneous by Proposition 1.1.

Conversely, assume that $X$ is neither an arc nor a simple closed curve. If every $\operatorname{arc}$ in $X$ is nowhere dense in $X$, then $C(X)$ is the Hilbert cube [IN, p. 89, 11.3] and, hence, is homogeneous [V, p. 254, 6.1.6]; thus, $C(X)$ is not $\frac{1}{2}$-homogeneous, and we are done.

So, assume that $X$ contains an $\operatorname{arc} A$ with nonempty topological interior $A^{\circ}$ in $X$.

Since $X$ is neither an arc nor a simple closed curve, $X$ contains a simple triod $T[\mathrm{~N} 2$, p. 135, 8.40(b)]. We consider three points of $C(X):\{p\}$ where $p \in A^{\circ}$, an $\operatorname{arc} B$ such that $B \subset A^{\circ}$ and $B \subset i A$, and $\{q\}$ where $q$ is the point of order 3 in $T$. We note that $C(A)$ is a 2-cell with $\{p\} \in \partial C(A)$ and $B \in i C(A)$ [IN, p. 33, 5.1.1], $C(A)$ is a neighborhood of both $\{p\}$ and $B$ in $C(X)$, and $\{q\}$ belongs to a 3 -cell in $C(T)$ [IN, p. 41, 5.4].

Hence, we have the following: $\{p\}$ does not belong to the manifold interior of a 2-cell in $C(X)$ (use [HW, p. 95, Theorem VI 9]); $B$ belongs to both the manifold interior and the topological interior in $C(X)$ of the 2-cell $C(A) ;\{q\}$ belongs to the manifold interior of a 2-cell in $C(X)$ and $\{q\}$ does not have a 2-cell neighborhood in $C(X)$ (use [HW, p. 95, Theorem VI9]).

Thus, the orbits $\mathcal{O}(\{p\}), \mathcal{O}(B)$ and $\mathcal{O}(\{q\})$ are mutually distinct. Therefore, $C(X)$ is not $\frac{1}{2}$-homogeneous.

The 2-fold hyperspace $C_{2}(X)$ of a continuum $X$ consists of all nonempty compact subsets of $X$ that have at most 2 components (with the Hausdorff metric). In the comment to Question 6.8, we prove that for locally connected continua $X, C_{2}(X)$ is $\frac{1}{2}$-homogeneous if and only if $X$ is an arc. 
The following lemma is well known but is not stated in the literature. Since the lemma may be useful in other connections, we state and prove the lemma here. Results in $[\mathrm{MN}]$ and $[\mathrm{N} 1]$ can be combined to prove the lemma; our proof is independent of results in [MN].

LEMMA 3.2. If $X$ is a hereditarily indecomposable continuum, then $C(X)$ is not connected im kleinen at any point $A \neq X$.

Proof. Let $A \in C(X)$ such that $A \neq X$, and let $\mathcal{N}$ be a closed, connected neighborhood of $A$ in $C(X)$. Let $a \in A$. Let $K_{a}$ denote the composant of $a$ in $X$, and let $K$ denote another composant of $X$ [N2, p. 203, 11.15]. We note that $K$ is dense in $X[\mathrm{~N} 2$, p. 83, 5.20(a)] and that $X$ has the property of Kelley at $a[\mathrm{~N} 1$, p. 553, 16.27]. Thus, since $a \in A \in \mathcal{N}$, there exist $b \in K$ and $B \in \mathcal{N}$ such that $b \in B$. Hence, $\cup \mathcal{N}$ is a subcontinum of $X[\mathrm{~N} 1$, p. 102, 1.49] that intersects each of the composants $K_{a}$ and $K$. Therefore, $\cup \mathcal{N}=X[\mathrm{~N} 2$, p. 204, 11.17]. This proves that $\mathcal{N}$ cannot be arbitrarily small since $A \neq X$.

LEMMA 3.3. If $X$ is a hereditarily indecomposable continuum, then $C(X)$ is not $\frac{1}{2}$-homogeneous.

Proof. Let $X$ be a nondegenerate hereditarily indecomposable continuum (the case when $X$ is degenerate is trivial). Let $p \in X$ and let $A$ be a nondegenerate proper subcontinuum of $X$. Note that $C(X)$ is arcwise connected [N1, p. 64, 1.12]. Hence, using the structure of arcs in $C(X)[\mathrm{N} 1$, p. 113, 1.62], it follows that $\{p\}$ does not belong to the manifold interior of any arc in $C(X)$ and that $A$ does belong to the manifold interior of an arc in $C(X)$. Furthermore, by Lemma 3.2, $C(X)$ is not connected im kleinen at $\{p\}$ or at $A$. Thus, since $C(X)$ is connected im kleinen at the point $X$ by 2.4 , $C(X)$ is not $\frac{1}{2}$-homogeneous.

LEMmA 3.4. Let $X$ be an atriodic continuum. If $C(X)$ is $\frac{1}{2}$-homogeneous, then all singletons are in the same orbit of $C(X)$.

Proof. By Lemma 3.3, $X$ contains a decomposable continuum $Y$. Then $C(Y)$ contains a 2 -cell $\Delta^{2}[\mathrm{~N} 1$, p. $156,1.145]$. Let $A \in i \Delta^{2}$. Then, by 2.11, no singleton is in the orbit $\mathcal{O}(A)$. Therefore, since $C(X)$ is $\frac{1}{2}$-homogeneous, all singletons are in the same orbit of $C(X)$.

Our next lemma reduces answering the question in Section 1, for the case of atriodic continua $X$, to proving that $X$ is connected im kleinen at one point. We use the lemma in the proof of Theorem 5.1.

LEMMA 3.5. Let $X$ be a nondegenerate atriodic continuum such that $X$ is connected im kleinen at some point $p$. Then $C(X)$ is $\frac{1}{2}$-homogeneous if and only if $X$ is an arc or a simple closed curve. 
Proof. Assume that $C(X)$ is $\frac{1}{2}$-homogeneous. Then, by Lemma 3.4, $C(X)$ is connected im kleinen at every singleton. Hence, $X$ is connected im kleinen at every point by 2.10 . Thus, $X$ is locally connected [N2, p. 84, $5.22(\mathrm{~b})]$. Therefore, by Theorem $3.1, X$ is an arc or a simple closed curve.

The converse is by Proposition 1.1.

The following lemma gives sufficient conditions for every element of $\mathcal{O}(X)$ to be a nondegenerate indecomposable continuum.

LEMMA 3.6. Let $X$ be a nonlocally connected atriodic continuum such that $C(X)$ is $\frac{1}{2}$-homogeneous. Assume that $Y$ is a nondegenerate indecomposable subcontinuum of $X$. Then $\mathcal{O}(X)=\mathcal{O}(Y)$; moreover, every element $A$ of $\mathcal{O}(X)$ is a nondegenerate indecomposable continuum and (equivalently) $C(X)-\{A\}$ has uncountably many arc components.

Proof. Since $X$ is not locally connected, $X$ is not connected im kleinen at some point $p[\mathrm{~N} 1, \mathrm{p} .83,5.22(\mathrm{~b})]$. Hence, $C(X)$ is not connected im kleinen at $\{p\}$ by 2.10 . Thus, by $2.4, \mathcal{O}(X) \neq \mathcal{O}(\{p\})$.

By 2.8, $C(X)-\{Y\}$ has uncountably many arc components. On the other hand, by 2.3, $C(X)-\{\{p\}\}$ is arcwise connected. Hence, $\mathcal{O}(Y) \neq \mathcal{O}(\{p\})$.

Since $C(X)$ is $\frac{1}{2}$-homogeneous, we can now conclude that $\mathcal{O}(Y)=\mathcal{O}(X)$.

Finally, let $A \in \mathcal{O}(X)$. Since $X \in \mathcal{O}(Y), C(X)-\{X\}$ has uncountably many arc components and, hence, so does $C(X)-\{A\}$. Therefore, $A$ is nondegenerate (since no singleton can arcwise disconnect $C(X)$ by 2.3). Furthermore, since $C(X)-C(A)$ is arcwise connected by $2.3, C(A)-\{A\}$ can not be arcwise connected; hence, $A$ is indecomposable by 2.5 (with $X=A$ ).

Our next lemma is the second key lemma used in the proof of Theorem 5.1. Regarding the proof of the lemma, see the acknowledgments at the end of the paper.

LEMMA 3.7. Let $X$ be a nonlocally connected atriodic continuum such that $C(X)$ is $\frac{1}{2}$-homogeneous. Then every nondegenerate proper subcontinuum of $X$ is decomposable.

Proof. Suppose by way of contradiction that $X$ has a nondegenerate indecomposable proper subcontinuum $Y$. Then, by Lemma 3.6, $Y \in \mathcal{O}(X)$. Hence, there is a homeomorphism $h$ of $C(X)$ onto $C(X)$ such that $h(Y)=X$. Clearly, $\mathcal{O}(Y)=\mathcal{O}(X)=\mathcal{O}(h(X))$. Therefore, by Lemma 3.6, $X, Y$ and $h(X)$ are nondegenerate indecomposable continua and each of $C(X)-\{Y\}$ and $C(X)-\{h(X)\}$ has uncountably many arc components. Hence, there is an arc component $\mathcal{A}$ of $C(X)-\{Y\}$ such that $X \notin \mathcal{A}$, and there is an arc component $\mathcal{B}$ of $C(X)-\{h(X)\}$ such that $X \notin \mathcal{B}$. Then, by 2.7 , there is a composant $K$ of $Y$ such that $\mathcal{A}=C(K)$, and there is a composant $M$ of $h(X)$ such that $\mathcal{B}=C(M)$. 
Note that $h(\mathcal{A})$ is an arc component of $h[C(X)-\{Y\}]=C(X)-\{X\}$. Also, $h^{-1}(\mathcal{B})$ is an arc component of $h^{-1}[C(X)-\{h(X)\}]=C(X)-\{X\}$. Hence, by 2.7 , there is a composant $J$ of $X$ such that $h(\mathcal{A})=C(J)$, and there is a composant $L$ of $X$ such that $h^{-1}(\mathcal{B})=C(L)$. Note that $L \cap Y=\emptyset$ (otherwise, since $Y$ is a proper subcontinuum of $X, Y \subset L$ and, hence, $Y \in$ $C(L)=h^{-1}(\mathcal{B})$; thus, $X \in \mathcal{B}$, a contradiction).

Finally, we prove (3.1) and (3.2) below which, taken together, contradict that $h$ is a homeomorphism.

$$
\overline{\mathcal{A}} \cap h^{-1}(\mathcal{B})=\emptyset .
$$

Proof of (3.1). Recall that $\mathcal{A}=C(K)$ and $K \subset Y$. Thus, since $C(Y)$ is compact we have that $\overline{\mathcal{A}} \subset C(Y)$. Also, since $h^{-1}(\mathcal{B})=C(L)$ and $L \cap Y=\emptyset$, we have that $h^{-1}(\mathcal{B}) \cap C(Y)=\emptyset$. Thus, (3.1) now follows.

$$
\overline{h(\mathcal{A})} \cap \mathcal{B} \neq \emptyset .
$$

Proof of $(3.2)$. Recall that $h(\mathcal{A})=C(J)$ and $\mathcal{B}=C(M)$. Hence, (3.2) is the same as

$$
\overline{C(J)} \cap C(M) \neq \emptyset .
$$

Let $p \in M$. Since $J$ is a composant of $X, \bar{J}=X[\mathrm{~N} 2$, p. 83, 5.20(a)]. Hence, there is a sequence $\left\{p_{n}\right\}_{n=1}^{\infty}$ in $J$ converging to $p$. Then $\left\{\left\{p_{n}\right\}\right\}_{n=1}^{\infty}$ is a sequence in $C(J)$ converging to $\{p\}$. Thus, $\{p\} \in \overline{C(J)}$. Therefore, since $\{p\} \in C(M)$, we have proved (3.3).

We now give information about how the decomposable proper subcontinua of $X$ are embedded in $X$ when $X$ is any continuum such that $C(X)$ is $\frac{1}{2}$ homogeneous. In connection with the theorem, recall Lemma 3.7 and see Theorem 3.11.

Theorem 3.8. Let $X$ be a continuum such that $C(X)$ is $\frac{1}{2}$-homogeneous. Then no decomposable proper subcontinuum of $X$ is terminal in $X$. 2.3 .

Proof. Let $p \in X$. Note that $C(X)-\{\{p\}\}$ is arcwise connected by

Assume that $T$ is a decomposable proper subcontinuum of $X$ such that $T$ is terminal in $X$.

By 2.9, $T$ arcwise disconnects $C(X)$; moreover, $C(X)-\{T\}$ has exactly two arc components (namely, $C(X)-C(T)$ by 2.3 and $C(T)-\{T\}$ by 2.5 since $T$ is decomposable). Hence, $\mathcal{O}(T) \neq \mathcal{O}(\{p\})$.

First, assume that $X$ is indecomposable. Then, since $X$ has uncountably many composants [N2, pp. 203, 11.15], $C(X)-\{X\}$ has uncountably many arc components [N1, pp. 105-106, 1.52.1(2)]. Hence, $\mathcal{O}(X) \neq \mathcal{O}(T)$ and $\mathcal{O}(X) \neq$ $\mathcal{O}(\{p\})$. Therefore, since $\mathcal{O}(T) \neq \mathcal{O}(\{p\}), C(X)$ is not $\frac{1}{2}$-homogeneous. 
Finally, assume that $X$ is decomposable. Then $C(X)-\{X\}$ is arcwise connected by 2.5 . Hence, $\mathcal{O}(X) \neq \mathcal{O}(T)$. Next, note that if $X$ were locally connected, then the only subcontinua of $X$ that would be terminal in $X$ are singletons (since locally connected continua are locally arcwise connected $[\mathrm{N} 2$, p. 131, 8.25]). Hence, by our assumptions about $T, X$ is not locally connected. Thus, we can assume we chose $p$ at the beginning of the proof so that $X$ is not connected im kleinen at $p$ [N1, p. 83, 5.22(b)]. Then $C(X)$ is not connected im kleinen at $\{p\}$ by 2.10 . Thus, by $2.4, \mathcal{O}(X) \neq \mathcal{O}(\{p\})$. Therefore, since $\mathcal{O}(T) \neq \mathcal{O}(\{p\})$ and $\mathcal{O}(X) \neq \mathcal{O}(T)$, we again have that $C(X)$ is not $\frac{1}{2}$-homogeneous.

Our remaining four results concern the situation when $C(X)$ is finite dimensional. Theorem 3.9 and Theorem 3.11, as well as their proofs, are similar to Lemma 3.6 and Lemma 3.7. We include the two theorems, as well as the others, because they provide information relevant to several questions in Section 6 (Questions 6.1, 6.2, 6.4 and 6.5).

THEOREM 3.9. Let $X$ be a nonlocally connected continuum such that $\operatorname{dim}(C(X))<\infty$ and $C(X)$ is $\frac{1}{2}$-homogeneous. Assume that $Y$ is a nondegenerate indecomposable subcontinuum of $X$. Then $\mathcal{O}(X)=\mathcal{O}(Y)$; moreover, every element $A$ of $\mathcal{O}(X)$ is a nondegenerate indecomposable continuum and (equivalently) $C(X)-\{A\}$ has uncountably many arc components.

Proof. The proof is the same as the proof of Lemma 3.6 using 2.6 in place of 2.8 .

Corollary 3.10. Let $X$ be a decomposable continuum such that $\operatorname{dim}(C(X))<\infty$. If $C(X)$ is $\frac{1}{2}$-homogeneous, then $X$ is hereditarily decomposable.

Proof. If $X$ is locally connected, apply Theorem 3.1. If $X$ is not locally connected, assume there is a nondegenerate indecomposable subcontinuum $Y$ of $X$; then apply Theorem 3.9 to obtain that $X$ is indecomposable.

THEOREM 3.11. Let $X$ be a nonlocally connected continuum such that $\operatorname{dim}(C(X))<\infty$ and $C(X)$ is $\frac{1}{2}$-homogeneous. Then every nondegenerate proper subcontinuum of $X$ is decomposable.

Proof. The proof is the same as the proof of Lemma 3.7 using Theorem 3.9 instead of Lemma 3.6.

Theorem 3.12. Let $X$ be any continuum such that $\operatorname{dim}(C(X))<\infty$. Then each nondegenerate subcontinuum of $X$ in the orbit $\mathcal{O}(\{p\})$ is decomposable for each $p \in X$. Hence, if $\operatorname{dim}(C(X))<\infty$ and $C(X)$ is $\frac{1}{2}$-homogeneous, and if all the singletons are not in the same orbit of $C(X)$, then $X$ is hereditarily decomposable. 
Proof. To prove the first part of the theorem, let $p \in X$ and let $A \in \mathcal{O}(\{p\})$ such that $A$ is nondegenerate. By 2.3, $C(X)-\{\{p\}\}$ is arcwise connected. Therefore, by 2.6, $A$ must be decomposable.

The second part of the theorem follows from the first part since, under the two additional assumptions for the second part, every point $A$ of $C(X)$ is in the orbit of some singleton.

We note that when all singletons are not in the same orbit of $C(X)$, as assumed in Theorem 3.12, then $X$ must contain triods by Lemma 3.4.

\section{A LEMMA ABOUt 2-CELLS IN HYPERSPACES}

We prove Lemma 4.2 for use in the next section. We use Lemma 4.1 in the proof of Lemma 4.2. The lemmas concern layers (finest monotone maps), which we discussed before stating 2.1. The implicit assumption in our lemmas that layers and finest monotone maps exist is guaranteed by 2.1 .

LEMMA 4.1. Let $Z$ be an irreducible hereditarily decomposable continuum, and let $\varphi: Z \rightarrow I$ be a finest monotone map. Let $Q \in C(Z)$ such that

$$
\varphi(Q)=[a, b] \text {, where } 0<a<b<1 \text {. }
$$

Fix $t_{0} \in(a, b)$. Then $\varphi^{-1}\left(t_{0}\right), Q \cap \varphi^{-1}\left(\left[a, t_{0}\right]\right)$ and $Q \cap \varphi^{-1}\left(\left[t_{0}, b\right]\right)$ are subcontinua of $Q$.

Proof. Since $a, b \in \varphi(Q)$ and $Z$ is irreducible from $\varphi^{-1}(0)$ to $\varphi^{-1}(1)$, we see that

$$
\varphi^{-1}([0, a]) \cup Q \cup \varphi^{-1}([b, 1])=Z .
$$

Hence,

$$
\varphi^{-1}((a, b)) \subset Q
$$

By (4.1), $\varphi^{-1}\left(t_{0}\right) \subset Q$ and, therefore, $\varphi^{-1}\left(t_{0}\right)$ is a subcontinnum of $Q$ since $\varphi$ is monotone.

To prove that $Q \cap \varphi^{-1}\left(\left[a, t_{0}\right]\right)$ and $Q \cap \varphi^{-1}\left(\left[t_{0}, b\right]\right)$ are subcontinua of $Q$, it is clear that we only need to prove that $Q \cap \varphi^{-1}\left(\left[a, t_{0}\right]\right)$ and $Q \cap \varphi^{-1}\left(\left[t_{0}, b\right]\right)$ are connected.

We prove that $Q \cap \varphi^{-1}\left(\left[a, t_{0}\right]\right)$ is connected; the proof that $Q \cap \varphi^{-1}\left(\left[t_{0}, b\right]\right)$ is connected is analogous. Let $A=\varphi^{-1}\left(\left(a, t_{0}\right]\right)$. Then $A$ is connected [N2, p. 137, 8.46], $A \subset Q$ by (4.1), and $Q \cap \varphi^{-1}(a)$ is both closed and open in $Q-A$. Thus, since $Q$ is connected, $\left(Q \cap \varphi^{-1}(a)\right) \cup A$ is connected [Du, p. 118, 2 under Section 3]. Therefore, since

$$
Q \cap \varphi^{-1}\left(\left[a, t_{0}\right]\right)=\left(Q \cap \varphi^{-1}(a)\right) \cup A,
$$

$Q \cap \varphi^{-1}\left(\left[a, t_{0}\right]\right)$ is connected. 
Lemma 4.2. Let $Z$ be an irreducible hereditarily decomposable continuum, and let $\varphi: Z \rightarrow I$ be a finest monotone map. Let $Q \in C(Z)$ such that

$$
\varphi(Q)=[a, b], \text { where } 0<a<b<1 .
$$

Then $Q$ belongs to the manifold interior of a 2 -cell in $C(Z)$.

Proof. Fix $t_{0} \in(a, b)$. Then, by Lemma 4.1, $\varphi^{-1}\left(t_{0}\right), Q \cap \varphi^{-1}\left(\left[a, t_{0}\right]\right)$ and $Q \cap \varphi^{-1}\left(\left[t_{0}, b\right]\right)$ are subcontinua of $Q$. Thus, by [IN, p. 112, 14.6], there is an order arc $\alpha_{1}$ in $C(Z)$ from $\varphi^{-1}\left(t_{0}\right)$ to $Q \cap \varphi^{-1}\left(\left[a, t_{0}\right]\right)$ and an order arc $\alpha_{2}$ in $C(Z)$ from $Q \cap \varphi^{-1}\left(\left[a, t_{0}\right]\right)$ to $\varphi^{-1}\left(\left[0, t_{0}\right]\right)$. Hence, $\alpha_{1} \cup \alpha_{2}=\alpha$ is an order arc in $C(Z)$ from $\varphi^{-1}\left(t_{0}\right)$ to $\varphi^{-1}\left(\left[0, t_{0}\right]\right)$ such that $Q \cap \varphi^{-1}\left(\left[a, t_{0}\right]\right) \in \alpha$. Similarly, there is an order arc $\beta$ in $C(Z)$ from $\varphi^{-1}\left(t_{0}\right)$ to $\varphi^{-1}\left(\left[t_{0}, 1\right]\right)$ such that $Q \cap \varphi^{-1}\left(\left[t_{0}, b\right]\right) \in \beta$.

Let $h_{1}$ and $h_{2}$ be homeomorphisms of $I$ onto $\alpha$ and $\beta$, respectively, such that $h_{1}(0)=h_{2}(0)=\varphi^{-1}\left(t_{0}\right)$. Define $h: I \times I \rightarrow C(Z)$ by

$$
h(s, t)=h_{1}(s) \cup h_{2}(t) \text { for all }(s, t) \in I \times I .
$$

Then $h$ is an embedding of the two cell $I \times I$ into $C(Z)$ (the continuity of $h$ follows using the continuity of the union map [IN, p. 9, 1.23]). Furthermore, $Q \in h((0,1) \times(0,1))$ and, therefore, $Q \in i h(I \times I)$.

\section{Main theOREM AND COROllaries}

We prove the principal result of our paper and note several corollaries.

THEOREM 5.1. Let $X$ be a nondegenerate atriodic continuum. Then $C(X)$ is $\frac{1}{2}$-homogeneous if and only if $X$ is an arc or a simple closed curve.

Proof. Assume that $C(X)$ is $\frac{1}{2}$-homogeneous. To prove that $X$ is an arc or a simple closed curve, it suffices by Lemma 3.5 to prove that $X$ is connected im kleinen at some point.

Suppose by way of contradiction that $X$ is not connected im kleinen at any point. Then, by $2.10, C(X)$ is not connected im kleinen at any singleton.

Let $y \in X$. Since $X$ is not connected im kleinen at $y$, there is an open neighborhood $U$ of $y$ in $X$ and a sequence $\left\{C_{n}\right\}_{n=1}^{\infty}$ of distinct components of $\bar{U}$ such that $\left\{C_{n}\right\}_{n=1}^{\infty}$ converges to a nondegenerate subcontinuum $C$ of $X$, where $y \in C$ and $C_{n} \cap C=\emptyset$ for all $n$ (this follows from the proof of Theorem 5.12 in $[\mathrm{N} 2$, pp. $76-78])$.

Note that $U \neq X$; hence, by $2.2, C_{n} \cap(\bar{U}-U) \neq \emptyset$ for each $n$. Therefore, $C \cap(\bar{U}-U) \neq \emptyset$. Thus, since $y \in C \cap U$, there is a nondegenerate continuum $K$ such that $y \in K \subset U \cap C$ (apply 2.2 to an open neighborhood of $y$ in $C$ whose closure lies in $U$ ). Let $Z$ be an irreducible subcontinuum of $K$. Note that $Z$ is hereditarily decomposable by Lemma 3.7 . Hence, by 2.1 , there is a finest monotone map $\varphi: Z \rightarrow I$. Let $q \in \varphi^{-1}\left(\frac{1}{2}\right)$. Note that $\varphi^{-1}\left(\frac{1}{2}\right) \subset Z \subset K \subset U$; 
hence, there is an open subset $V$ of $X$ such that

$$
\varphi^{-1}\left(\frac{1}{2}\right) \subset V \subset \bar{V} \subset U \text { and } \bar{V} \cap \varphi^{-1}(\{0,1\})=\emptyset .
$$

Since $q \in Z \subset K \subset C$ and the sequence $\left\{C_{n}\right\}_{n=1}^{\infty}$ converges to $C$, there is a sequence $\left\{q_{n}\right\}_{n=1}^{\infty}$ converging to $q$ such that $q_{n} \in C_{n}$ for each $n$. Since $q \in V$, we can assume that $q_{n} \in V$ for each $n$. Then, for each $n$, the component $Q_{n}$ of $\overline{C_{n} \cap V}$ containing $q_{n}$ exists. Without loss of generality, we assume that the sequence $\left\{Q_{n}\right\}_{n=1}^{\infty}$ converges to a continuum $Q[\mathrm{~N} 1, \mathrm{p} .7,0.8]$. Hence,

$$
q \in Q \subset C \cap \bar{V} \subset U .
$$

Since $V \subset U$ by (5.1) and $C_{n} \cap(\bar{U}-U) \neq \emptyset$ for each $n$, we have that $C_{n} \cap V \neq C_{n}$; hence, by $2.2, Q_{n} \cap(\bar{V}-V) \neq \emptyset$ for each $n$. Thus, since $\lim _{n \rightarrow \infty} Q_{n}=Q$, we see that

$$
Q \cap(\bar{V}-V) \neq \emptyset .
$$

Let $i \neq j$. Since $C_{i}$ and $C_{j}$ are different components of $\bar{U}$, and since $Q_{i} \subset C_{i} \cap \bar{V} \subset U$ and $Q_{j} \subset C_{j} \cap \bar{V} \subset U$, it follows that $Q_{i}$ and $Q_{j}$ are contained in different components of $U$. Therefore, since $Q \subset U$ by (5.2) and $Q=\lim _{n \rightarrow \infty} Q_{n}$, we have by [N1, p. 156, 1.143] that

$$
C(X) \text { is not connected im kleinen at } Q \text {. }
$$

Next, we prove that $Q \subset Z$. We use the fact that since $X$ is atriodic, $X$ does not contain a weak triod [S, p. 443, Theorem 1.8]. Let

$$
W=\varphi^{-1}\left(\left[0, \frac{1}{2}\right]\right) \cup Q \cup \varphi^{-1}\left(\left[\frac{1}{2}, 1\right]\right) .
$$

We chose $q \in \varphi^{-1}\left(\frac{1}{2}\right)$; thus, since $q \in Q$ by (5.2),

$$
\varphi^{-1}\left(\left[0, \frac{1}{2}\right]\right) \cap Q \cap \varphi^{-1}\left(\left[\frac{1}{2}, 1\right]\right) \neq \emptyset .
$$

By (5.2), $Q \subset \bar{V}$; hence, by (5.1), $Q \cap \varphi^{-1}(\{0,1\})=\emptyset$. This shows that $\varphi^{-1}\left(\left[0, \frac{1}{2}\right]\right) \not \subset Q \cup \varphi^{-1}\left(\left[\frac{1}{2}, 1\right]\right)$ and $\varphi^{-1}\left(\left[\frac{1}{2}, 1\right]\right) \not \subset Q \cup \varphi^{-1}\left(\left[0, \frac{1}{2}\right]\right)$. Therefore, since $W$ is not a weak triod, $Q \subset Z$.

By assumption, $C(X)$ is $\frac{1}{2}$-homogeneous. Let $\mathcal{O}_{1}$ and $\mathcal{O}_{2}$ denote the two orbits of $C(X)$. We now proceed to obtain a contradiction.

We assume without loss of generality that $Q \in \mathcal{O}_{1}$.

For the purpose of applying Lemma 4.2, we note the following: First, $Q \cap \varphi^{-1}\left(\frac{1}{2}\right) \neq \emptyset$ since $q \in Q$ by (5.2); second, $Q \not \subset \varphi^{-1}\left(\frac{1}{2}\right)$ since $\varphi^{-1}\left(\frac{1}{2}\right) \subset V$ by $(5.1)$ and $Q \cap(\bar{V}-V) \neq \emptyset$ by (5.3); third, $Q \cap \varphi^{-1}(\{0,1\})=\emptyset$ by $(5.1)$ since $Q \subset \bar{V}$ by (5.2). Thus, since $Q \subset Z$,

$$
\varphi(Q)=[a, b], \text { where } 0<a<b<1 .
$$

Therefore, by Lemma 4.2, we have that

$Q$ belongs to the manifold interior of a 2-cell in $C(X)$. 
Since $Q \in \mathcal{O}_{1}$, we have by (5.5) and 2.11 that every singleton belongs to $\mathcal{O}_{2}$. Thus, since $C(X)$ is not connected im kleinen at singletons (as noted near the beginning of the proof), $C(X)$ is not connected im kleinen at any element of $\mathcal{O}_{2}$. Also, since $Q \in \mathcal{O}_{1}$, we have by $(5.4), C(X)$ is not connected im kleinen

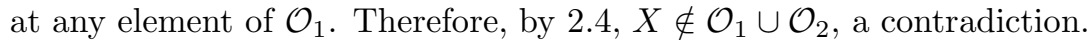

The other direction of our theorem is by Proposition 1.1.

We give several corollaries to Theorem 5.1. The corollaries concern various classes of continua that are of significant interest in hyperspace theory as well as continuum theory.

The class of continua all of whose nondegenerate proper subcontinua are arc-like has been widely investigated. We note the following corollary:

Corollary 5.2. Let $X$ be a continuum such that each nondegenerate proper subcontinuum of $X$ is arc-like. Then $C(X)$ is $\frac{1}{2}$-homogeneous if and only if $X$ is an arc or a simple closed curve.

Proof. The continuum $X$ is atriodic (as follows easily from [N2, p. 231, 12.4] using 2.2). Thus, the corollary follows from Theorem 5.1.

The next three corollaries are special cases of Corollary 5.2.

Corollary 5.3. If $X$ is an arc-like continuum, then $C(X)$ is $\frac{1}{2}$ homogeneous if and only if $X$ is an arc.

Corollary 5.4. If $X$ is a circle-like continuum, then $C(X)$ is $\frac{1}{2}$ homogeneous if and only if $X$ is a simple closed curve.

Corollary 5.5. If $X$ is a tree-like continuum all of whose nondegenerate proper subcontinua are arc-like, then $C(X)$ is $\frac{1}{2}$-homogeneous if and only if $X$ is an arc.

Corollary 5.6. If $X$ is a nondegenerate atriodic tree-like continuum, then $C(X)$ is $\frac{1}{2}$-homogeneous if and only if $X$ is an arc.

Proof. Apply Theorem 5.1.

Corollary 5.6 shows, for example, that the hyperspaces $C(X)$ of the continua $X$ constructed in [DI] and [In] are not $\frac{1}{2}$-homogeneous.

A continuum all of whose nondegenerate proper subcontinua are arcs is called an arc-continuum [IN, p. 245]. For example, the solenoids are arccontinua, as is the continuum in [DI]. Arc-continua and their hyperspaces have been widely studied. Obviously, Theorem 5.1 applies to any arc-continuum.

Our final corollary is less specific than the others.

Corollary 5.7. Let $X$ be a continuum such that $\operatorname{dim}(C(X))=2$. Then $C(X)$ is $\frac{1}{2}$-homogeneous if and only if $X$ is an arc or a simple closed curve. 
Proof. Since $\operatorname{dim}(C(X))=2, X$ is atriodic $[N 1$, p. 140, 1.100]. Therefore, the corollary follows from Theorem 5.1.

We note that Corollary 5.2 follows from Corollary 5.7 using [N1, pp. 212 and 223, 2.1 and 2.14]. Regarding Corollary 5.7, see Question 6.2.

\section{Questions}

We raise questions that are directly related to what we have done. We include comments with the questions.

Our first two questions are directly connected with the general question in Section 1.

QUESTION 6.1. If $X$ is a continuum such that $C(X)$ is $\frac{1}{2}$-homogeneous, then are all singletons in the same orbit of $C(X)$ ?

Comment. Obviously, a negative answer would provide a negative answer to the question in Section 1. For a result that assumes all singletons are not in the same orbit of $C(X)$, see Theorem 3.12.

Question 6.2. If $X$ is a continuum such that $C(X)$ is $\frac{1}{2}$-homogeneous, then is $\operatorname{dim}(C(X))<\infty$ ? In fact, is $\operatorname{dim}(C(X))=2$ ?

Comment. An affirmative answer to the second part of the question would give an affirmative answer to the question in Section 1 by Corollary 5.7. We do not know the answer to the second part of the question under the assumption that $\operatorname{dim}(C(X))<\infty$.

Our next question focuses on a special case of the question in Section 1.

QUESTION 6.3. If $X$ is a tree-like continuum and $C(X)$ is $\frac{1}{2}$-homogeneous, then is $X$ an arc? What about when $\operatorname{dim}(C(X))<\infty$ ?

Comment. The question asks if the assumptions about tree-like continua in Corollary 5.5 and Corollary 5.6 can be omitted or at least replaced by the assumption that $\operatorname{dim}(C(X))<\infty$.

For our next two questions, recall that we gave sufficient conditions in Corollary 3.10 and Theorem 3.12 for $X$ to be hereditarily decomposable.

Question 6.4. If $X$ is a continuum such that $C(X)$ is $\frac{1}{2}$-homogeneous, then is $X$ decomposable? What about when $\operatorname{dim}(C(X))<\infty$ ?

Comment. By Lemma 3.3, X can not be hereditarily indecomposable.

An affirmative answer to the second part of the question would imply that $X$ is hereditarily decomposable (Corollary 3.10). Theorem 3.12 gives a partial answer to the second part of the question.

Question 6.5. If $X$ is a continuum such that $C(X)$ is $\frac{1}{2}$-homogeneous, then is $\operatorname{dim}(X)=1$ ? In fact, must $X$ be hereditarily decomposable? Must $X$ be hereditarily decomposable when $\operatorname{dim}(C(X))<\infty$ ? 
Comment. To answer the first part of the question affirmatively it is sufficient to prove that $\operatorname{dim}(C(X))<\infty$ [IN, pp. 348 and 354, 72.5 and 73.9]. Also, even though we know from Lemma 3.3 that $X$ can not be hereditarily indecomposable, we do not know if $X$ can contain a nondegenerate hereditarily indecomposable continuum - if it does not, then $\operatorname{dim}(X)=1$ [B, p. 270, Theorem 5]. The second part of Theorem 3.12 gives conditions under which $X$ is hereditarily decomposable, hence $\operatorname{dim}(X)=1$ [N2, p. 305, 13.57].

An affirmative answer to the second part of the question yields an affirmative answer to the first part of the question [N2, p. 305, 13.57]. Corollary 3.10 provides an affirmative answer to the second part of the question when $X$ is decomposable and $\operatorname{dim}(C(X))<\infty$.

Information that may be helpful in answering the third part of the question is in Theorem 3.9 through Theorem 3.12.

Our next two questions are related to 2.11, which was a key ingredient in the proof of Theorem 5.1. Thus, answers to the questions would probably be helpful in answering the question in Section 1 (and Question 6.3).

QUeSTION 6.6. What conditions on continua $X$ (other than being atriodic) or on points $p \in X$ are necessary and/or sufficient for $\{p\}$ not to belong to the manifold interior of a 2-cell in $C(X)$ ?

Comment. One might suspect that for locally connected continua $X$, a point $p$ is as in the question if $p$ is of order $\leq 2$ in $X$. However, let $X$ be the dendrite in the plane that is the union of the segment $S=I \times\{0\}$ and the segments $\left\{\frac{1}{n}\right\} \times\left[0, \frac{1}{n}\right]$, and let $p=(0,0)$; then $p$ is of order 1 in $X$, but $\{p\}$ belongs to the manifold interior of an $n$-cell in $C(X)$ for every $n$ since the space $C_{p}(X)$ of all subcontinua of $X$ that contain $p$ is the Hilbert cube [IN, p. 50,6.4].

On the other hand, if $\operatorname{dim}(C(X))<\infty$ and, as before, $X$ is a locally connected continuum, a point $p$ is as in Question 6.6 if and only if $p$ is of order $\leq 2$ in $X$. This follows from the fact that $X$ is a finite graph ([IN, p. 347, 72.2] or [N1, p. 144, 1.109]) and from models for $C(Y)$ when $Y$ is an arc, a simple closed curve or a simple triod [IN, pp. 33-43].

Our comments suggest that it might be a good idea to start investigating Question 6.6 for continua $X$ such that $\operatorname{dim}(C(X))<\infty$. We remark that one can consider Question 6.6 for $n$-cells in general.

Question 6.7. Is there a continuum $X$ such that $\operatorname{dim}(C(X))<\infty$ and, for every $x \in X,\{x\}$ is a point of the manifold interior of a 2-cell in $C(X)$ ?

Several special hyperspaces other than $C(X)$ are of general interest - the hyperspace $2^{X}$ of all nonempty compact subsets of a continuum $X$ (with the Hausdorff metric), the $n$-fold hyperspace $C_{n}(X)$ of all elements of $2^{X}$ with at most $n$ components, and the $n$-fold symmetric product $F_{n}(X)$ of all elements of $2^{X}$ with at most $n$ points. Our final question concerns these hyperspaces. 
Question 6.8. For what continua $X$ are the hyperspaces $2^{X}, C_{n}(X)$ or $F_{n}(X) \frac{1}{2}$-homogeneous $\left(n>1\right.$ for the case of $\left.F_{n}(X)\right)$ ? What about $\frac{1}{m}$ homogeneity for any integer $m>1$ ?

Comment. For $C_{1}(X)$, the first part of the question is a less specific form of the question in Section 1 since $C_{1}(X)=C(X)$. The second part of the question includes the evident question: when is $C(X) \frac{1}{m}$-homogeneous for various integers $m>1$ ?

The case of $\frac{1}{2}$-homogeneity for $C_{2}(X)$ seems especially interesting: $C_{2}(I)$ is $\frac{1}{2}$-homogeneous since $C_{2}(I)$ is a 4 -cell ([I1, p. 349, Lemma 2.2], due to R. M. Schori); however, $C_{2}\left(S^{1}\right)$ is not $\frac{1}{2}$-homogeneous since $C_{2}\left(S^{1}\right)$ is the cone over a solid torus [I2]. This (naively) suggests that $C_{2}(X)$ may only be $\frac{1}{2}$-homogeneous when $X$ is an arc. In fact, this is true when $X$ is locally connected (modify the proof of Theorem 3.1 as follows: $\{p\}$ is as in the proof, replace $B$ with $B_{1} \cup B_{2}$ where $B_{1}$ and $B_{2}$ are disjoint subarcs of $A^{\circ}$, replace $q$ with $\{p, q\}(\{p, q\}$ then belongs to a 5 -cell, not just a 3 -cell), and change statements about 2-cells to statements about 4-cells).

The hyperspace $2^{X}$ can not be $\frac{1}{2}$-homogeneous for any locally connected continuum $X$ since $2^{X}$ is the Hilbert cube (assuming $X$ is nondegenerate) [IN, p. 89, 11.3].

ACKNOWLEDGEMENTS.

We are grateful to the referee. We had originally proved several versions of Theorem 5.1 with additional assumptions. The referee found a way to use ideas in our original manuscript to obtain Theorem 3.11, which led us to be able to formulate and prove Lemma 3.7 and, thus, to prove Theorem 5.1 as stated here.

We thank Professor Gerardo Acosta for calling 2.11 to our attention. Our original proof of Corollary 5.2 was preceded by a special case of 2.11 whose argument was somewhat involved.

The second author expresses gratitude to DGAPA, UNAM, for supporting her visit to West Virginia University, where this paper was written.

\section{REFERENCES}

[A] G. Acosta, Continua with unique hyperspace, Continuum Theory, Lecture Notes in Pure and Applied Mathematics 230, Marcel Dekker, Inc., New York and Basel, 2002.

[B] R. H. Bing, Higher-dimensional hereditarily indecomposable continua, Trans. Amer. Math. Soc. 71 (1951), 267-273.

[DI] J. F. Davis and W. T. Ingram, An atriodic tree-like continuum with positive span which admits a monotone mapping to a chainable continuum, Fund. Math. 131 (1988), 13-24.

[Du] J. Dugundji, Topology, Allyn and Bacon, Inc., Boston, 1966.

[G] J. T. Goodykoontz, Jr., Connectedness im kleinen and local connectedness in $2^{X}$ and $C(X)$, Pacific J. Math. 53 (1974), 387-397.

[HW] W. Hurewicz and H. Wallman, Dimension Theory, Princeton University Press, Princeton, N. J., 1948 (revised edition). 
[I1] A. Illanes, The hyperspace $C_{2}(X)$ for a finite graph $X$ is unique, Glasnik Mat. 37 (2002), $347-363$.

[I2] A. Illanes, $A$ model for the hyperspace $C_{2}\left(S^{1}\right)$, Questions and Answers Gen. Topology 22 (2004), 117-130.

[IN] A. Illanes and S. B. Nadler, Jr., Hyperspaces: Fundamentals and Recent Advances, Pure and Applied Mathematics Series 216, Marcel Dekker, Inc., New York and Basel, 1999.

[In] W. T. Ingram, An atriodic tree-like continuum with positive span, Fund. Math. $\mathbf{7 7}$ (1972), 99-107.

[Kr] J. Krasinkiewicz, On homeomorphisms of the Sierpiński curve, Comment. Math. 12 (1969), 255-257.

[Ku] K. Kuratowski, Topology, Vol. II, Academic Press, N. Y., 1968.

[MN] S. Macías and S. B. Nadler, Jr., Various types of local connectedness in $n$-fold hyperspaces, Topology Appl., to appear.

[N1] S. B. Nadler, Jr., Hyperspaces of Sets - A Text with Research Questions, Pure and Applied Mathematics Series, Vol. 49, Marcel Dekker, Inc., New York and Basel, 1978.

[N2] S. B. Nadler, Jr., Continuum Theory: An Introduction, Pure and Applied Mathematics Series, Vol. 158, Marcel Dekker, Inc., New York, Basel and Hong Kong, 1992.

[P] H. Patkowska, On $\frac{1}{2}$-homogeneous ANR-spaces, Fund. Math. 132 (1989), 25-58.

[R] J. T. Rogers, Jr., Continua with cones homeomorphic to hyperspaces, General Topology and Appl. 3 (1973), 283-289.

[S] R. H. Sorgenfrey, Concerning triodic continua, Amer. J. Math. 66 (1944), 439-460.

[T] E. S. Thomas, Jr., Monotone decompositions of irreducible continua, Dissertationes Math. (Rozprawy Mat.) 50 (1966), 1-74.

[V] J. van Mill, Infinite-dimensional Topology: Prerequisites and Introduction, NorthHolland and Elsevier Sci. Publ. Co., Inc., Amsterdam, 1988.

S .B. Nadler, Jr.

Department of Mathematics

West Virginia University

P. O. Box 6310

Morgantown, WV 26506-6310

USA

E-mail: nadler@math.wvu.edu

P. Pellicer-Covarrubias

Department of Mathematics

West Virginia University

P. O. Box 6310

Morgantown, WV 26506-6310

USA

Departamento de Matemáticas

Facultad de Ciencias

Circuito Exterior

\& Ciudad Universitaria

México, D. F., C. P. 04510

México

E-mail: paty@ciencias.unam.mx \& paty@math.wvu.edu

Received: 31.1 .2005$.

Revised: 11.4.2005. \& 1.6.2005. 